\title{
Acoustic Emission during Mechanical Deformation of Polycarbonate
}

\author{
Shigenori ShIRouzU,* Shiro ShIChIJYo, Seiji TAKI, \\ Kazumi Matsushige, Kiyoshi TAKAHASHI, ${ }^{* *}$ \\ and Tetuo TAKEMURA \\ Department of Applied Science, Faculty of Engineering, \\ Kyushu University, Hakozaki, Fukuoka 812, Japan \\ ** Research Institute for Applied Mechanics, Kyushu University, \\ Kasuga, Kasuga-shi, Fukuoka 816, Japan
}

(Received November 7, 1983)

\begin{abstract}
An acoustic emission (AE) measurement was performed on a typical ductile polymer, polycarbonate (PC), during mechanical deformation, using a position detective AE measuring system. To find a correlation between the AE and mechanical deformation process, several AE characteristics, such as a wave form, duration time of AE signals and distribution of AE sources in the tensile specimen, were analyzed. The results suggest that there are two different mechanisms for AE occurrence in PC, one probably generated upon submicrocrack formation and the other, associated with molecular chain slipping at the necking front.
\end{abstract}
KEY WORDS Acoustic Emission / Mechanical Deformation /
Polycarbonate / Ductile / Submicrocrack / Necking / Small Angle X-Ray Scattering /

Acoustic emission consists of transient stress waves, generated by the partial rapid release of energy stored in material during mechanical deformation. By detecting and analyzing AE signals, the microscopic and instantaneous mechanism of the deformation process can be estimated. Thus, this AE method has been widely applied to the investigation of fracture processes in metallic materials and ceramics.

However, its application to polymeric materials is rather limited. ${ }^{1-9}$ Since AE signals in polymeric materials are very weak owing to the low released elastic energy and large attenuation in frequency range of AE signal. When a high gain $\mathrm{AE}$ detection system was used to detect such weak AE signals, the true signals became obscured among various noises, produced by the tensile machine, slipping noise from the sample gripping part, and electromagnetic noise from the outside. Therefore, the main problem in the application of
AE method to polymeric materials lies in the complete exclusion or discrimination of these noises from intrinsic AE signals.

Using a simple ring-down counting method, Grabec and Peterlin ${ }^{2}$ investigated the acoustic activity of polyvinyltoluene under tensile loading and bending, and reported the occurrence of a large number of $\mathrm{AE}$ events. However, in their measuring system, it is doubtful whether all noises were sufficiently excluded. Employing a noiseless testing machine using air bearings in all mobile parts, Betteridge et $a l^{7}$ reported a rather small number of $\mathrm{AE}$ events in several polymers. Even with this system, however, slipping noise at sample grips and the surface of AE sensors could not be avoided. Thus, the AE data so far reported can not be accepted entirely as intrinsic and reliable.

For more reliable AE data, the use of a position detective apparatus which can exclude

\footnotetext{
* Present address: Idemitsu-Kosan, Sodegaura, Chiba 292-01, Japan.
} 


\section{S. SHIROUZu et al.}

various noises by monitoring source location is necessary. Recently, with a position detective AE observation system, we performed AE measurements on poly(methyl methacrylate) during tensile deformation. ${ }^{1}$ Summarizing the AE results with Brillouin scattering, small angle $\mathrm{X}$-ray scattering and ultrasonic measurements, we obtained that embryonic stages for submicrocracks exist at a low level of tensile stress and $\mathrm{AE}$ waves are generated when these embryos develope submicrocracks. The detection of the generation of submicrocracks by the $\mathrm{AE}$ method is new.

Other aspects of the application of AE to the mechanical deformation of polymers were also considered. Most of previous investigations were conducted to detect the craze formation in brittle polymers, ${ }^{1-6}$ but only a few studies have been performed on ductile polymers. ${ }^{7-9}$ A large deformation process such as necking formation and propagation is an unexplored area in AE studies.

In this study, AE measurement was performed during tensile deformation for a typi- cal ductile polymer, polycarbonate, and the $\mathrm{AE}$ characteristics of individual $\mathrm{AE}$ events, such as wave form and the source location were investigated to find a correlation between these AE characteristics and the deformation mechanism.

\section{EXPERIMENTAL}

Tested samples were cut from a sheet of commercially-available PC polymer (Takiron Co., Takiron PC-4600) in the shape of a dumbbell $(180 \mathrm{~mm}$ in total length; $80 \mathrm{~mm}$ in total width; $10 \mathrm{~mm}$ in gauge length; $10 \mathrm{~mm}$ in gauge width, and $5 \mathrm{~mm}$ in thickness, respectively). The sample geometry was designed so as to have relatively short gauge length and long total length to reduce the damping of $\mathrm{AE}$ signals and enhance the damping of noises from the machine and/or clamping parts as much as possible. To minimize the frictional noise generated at the clamps, a pin-chuck was used as a sample grip.

The block diagram of the position detective

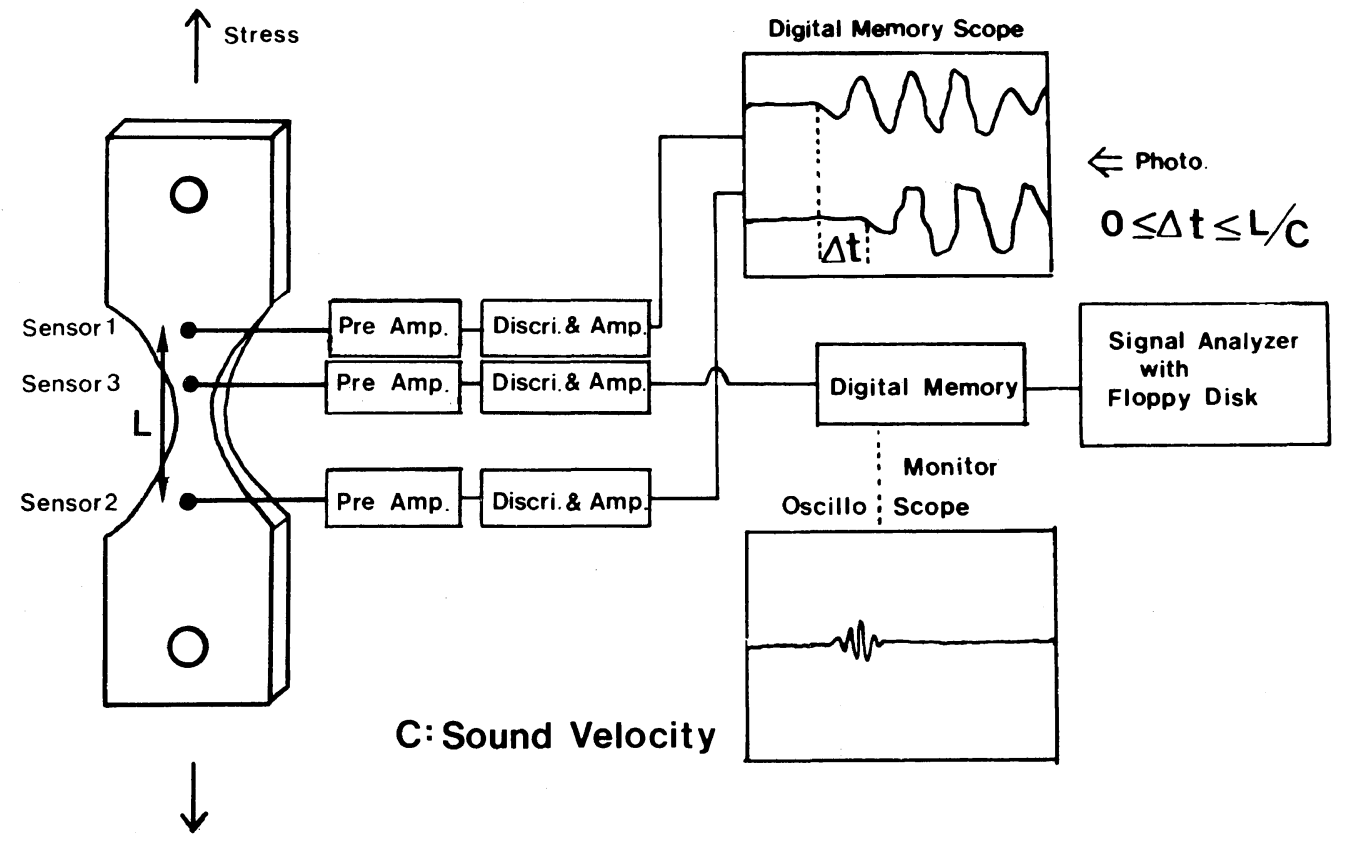

Figure 1. Block diagram of the AE measuring system. 
AE observing system used in this study is shown in Figure 1. Tensile load was applied to the specimen with a tensile machine (Shimadzu DSS-5000, cross-head speed; $1 \mathrm{~mm} \mathrm{~min}^{-1}$ ), and AE signals were simultaneously detected with three sensors acoustically coupled to a specimen by silicone grease. Two of the sensors (NF AE-901D, resonance frequency; $140 \mathrm{kHz}$ ) were set symmetrically at equivalent distances from the center of the gauge region and designated as sensor 1 and sensor 2. The third sensor (sensor 3 ) having a comparatively flat frequency characteristics (AET Co. MC-500) and low sensitivity was set near the gauge region. Total amplification in the $\mathrm{AE}$ signal detection was $90 \mathrm{~dB}$ for AE-901D and 100dB for MC-500, respectively. Signals detected with sensors 1 and 2 were displayed on a digital memory-scope (Iwatsu, DMS-6430) and recorded by photographs. The analysis of the location of the AE source was performed by observing the difference in arrival times of the AE signals at these sensors, $\Delta t$. When $\Delta t$ was larger than $L / c$, it was judged as the noise propagated from the outside of the gauge region, where $L$ is the distance between sensors 1 and 2 and $c$, the sound velocity of the specimen, respectively. On the other hand, signals detected by sensor 3 were recorded on a digital memory (Riken, TCF-2000), transported to a signal analyzer (Iwatsu, SM$2100 \mathrm{~A}$ ), and then collected in floppy disks. From these data, noise was excluded on the basis of the arrival time described for sensors 1 and 2. By these procedures it was possible to pick up only the intrinsic AE signals and guarantee reliable results.

At the same time, optical photographs of the samples were taken during deformation to find a correlation between the location of the $\mathrm{AE}$ source and deformation process.

To establish a relation between the $\mathrm{AE}$ events and occurrence of submicrocracks, small angle X-ray scattering (SAXS) measurements for thin film (thickness $1 \mathrm{~mm}$ ) specimen were performed. Details of the measuring sys- tem for SAXS is described elsewhere. ${ }^{1}$

\section{RESULTS}

Figure 2 shows the typical distribution of AE signals, considered as the true signals obtained by the AE measuring system, and observed stress-strain curve. The deformation process was divided for convenience into the following four stages: (1) linear region where the stress-strain curve is a straight line, (2) pre-necking stage where stress-strain curve deviates from a linear relation, (3) necking formation stage containing the yield region and (4) necking propagation stage. AE signals were observed throughout the entire stage of deformation. The marks appearing on the stress-strain curve show the points at which $\mathrm{AE}$ events were detected, but their height is not always proportional to the amplitude of the signals. Many other experiments have shown similar AE signals, assurring their reproducibility. The AE events indicated by dotted lines in the figure were very weak in amplitude and observed only under ideal measuring conditions.

To find the deformation processes correlated to $\mathrm{AE}$ events, $\mathrm{AE}$ characteristics such as wave form and distribution of emission point (where AE signals are generated in the specimen), were investigated at each stage of deformation. Figure 3 shows typical wave

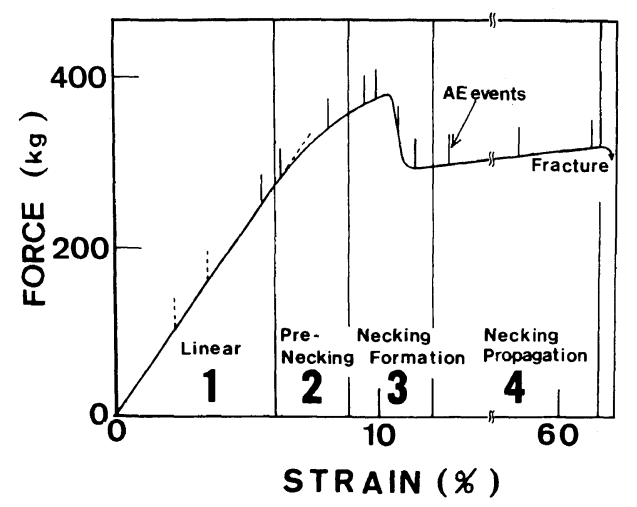

Figure 2. Typical distribution of AE events and stressstrain curve. 


\section{TYPICAL WAVE FORMS}
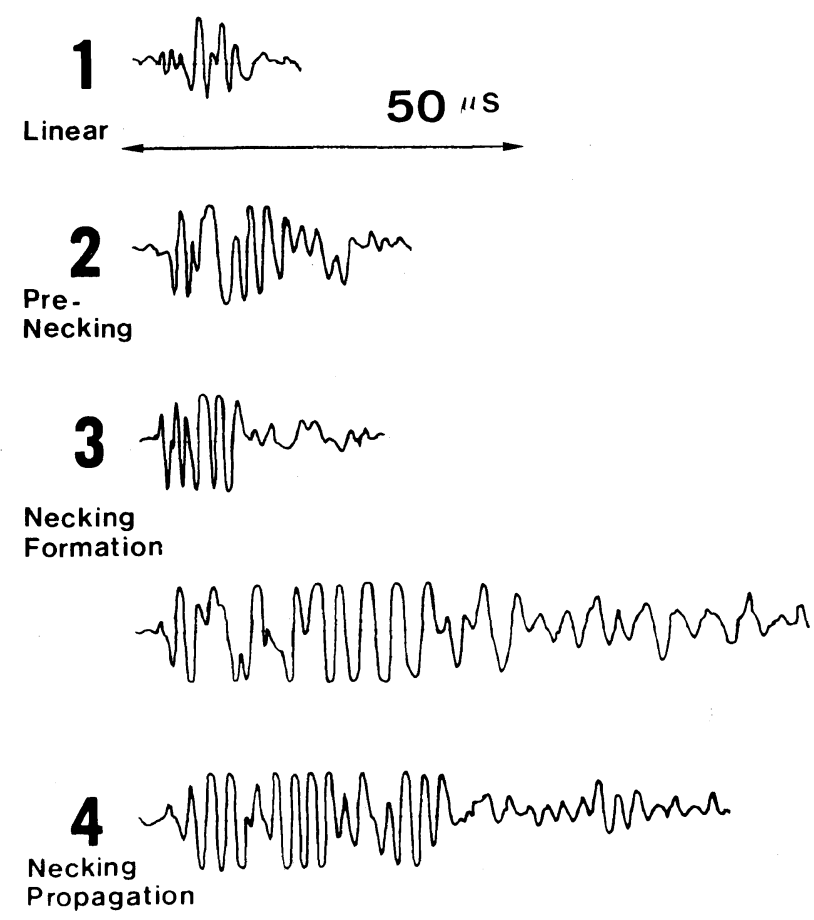

Figure 3. Typical wave forms at different deformation stages.

forms of AE signals observed at each such stage. The wave forms change remarkably on necking and clearly two kinds of AE signals exist. The differences in wave forms are evident on the basis of the duration time, $\tau$. Before necking formation (stages 1,2 ) the duration time is shorter than $50 \mu \mathrm{s}$ and after the formation (stage 4) longer than $80 \mu \mathrm{s}$. Between these two stages, i.e., (stage 3) both AE signals could be detected. Duration time distribution is summarized in Table $I$.

For a better understanding of the correlation between emission points and each deformation stage the distribution of these points was investigated throughout all stages. As shown in the lower column of Table I, distribution was evaluated by the value of $\sigma^{2}$, the mean square distance of emission points from certain reference points in the specimen. The reference points were chosen as a center line of the gauge region before the necking formation,
Table I. AE characteristics at each stage of deformation

\begin{tabular}{lcccc}
\hline & $\begin{array}{c}\text { Linear } \\
\text { Pre- }\end{array}$ & $\begin{array}{c}\text { necking } \\
\text { necking }\end{array}$ & $\begin{array}{c}\text { Necking } \\
\text { formation } \\
\text { propagation }\end{array}$ \\
\hline $\begin{array}{c}\text { Duration } \\
\text { time }(\mu \mathrm{s})\end{array}$ & $16 \sim 20$ & $25 \sim 50$ & $25 \sim 80$ & $80 \sim$ \\
\hline$\sigma^{2}\left(\mathrm{~mm}^{2}\right)$ & 6.3 & 3.7 & 3.8 & 1.9 \\
\hline
\end{tabular}

while the necking front (the portion where necking develops to the unnecked region and which is determined simultaneously by optical observation) after the necking formation. The values of $\sigma^{2}$ decreased from $6.0\left(\mathrm{~mm}^{2}\right)$ in the linear region (1) to $1.9\left(\mathrm{~mm}^{2}\right)$ at the necking propagation stage (4) with mechanical deformation. Another notable point is that at the pre-necking stage (2) where no macroscopic dimensional change could be admitted optically, the $\sigma^{2}$ values were relatively low. 


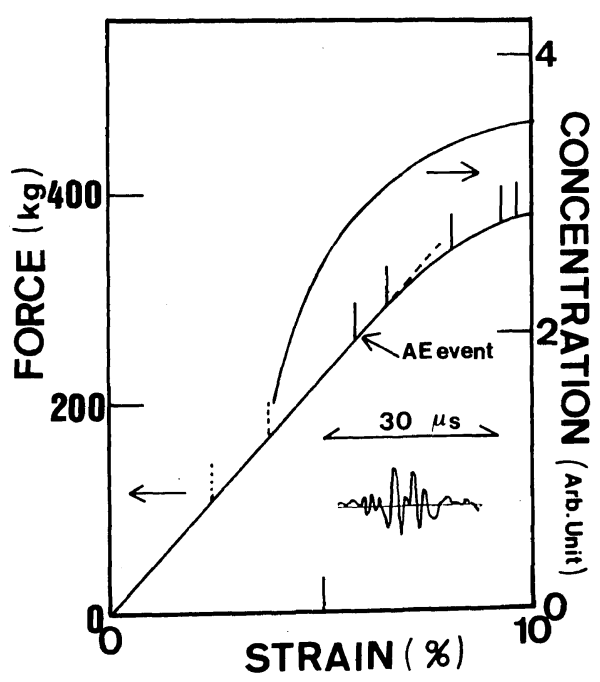

Figure 4. Strain dependence of concentration of submicrocracks and AE events on stress-strain curve before necking formation.

To observe the formation of submicrocracks, SAXS measurements were conducted. Diffuse X-ray scattering due to nucleation of the submicrocracks was observed at the strained stage above $\varepsilon, 3-4 \%$. Figure 4 shows the strain dependence of submicrocrack concentration along with $\mathrm{AE}$ events on the stress-strain curve up to the yield point.

\section{DISCUSSION}

A typical ductile amorphous polymer, PC, was found to generate many strong burst type AE signals throughout mechanical deformation. Furthermore, the $\mathrm{AE}$ signals in $\mathrm{PC}$ showed remarkable changes in wave form and emission point distribution, before and after necking formation. Thus, at least two mechanisms of acoustic emission may exist.

Before the necking formation (stage 1), the duration time of $\mathrm{AE}$ signals was less than $50 \mu \mathrm{s}$, and the value of $\sigma^{2}$ relatively high. The low value of $\tau$ suggests that the AE signals were generated by a rapid release of energy, while high value of $\sigma^{2}$ suggests that they occurred rather randomly throughout the gauge length. The value of $\sigma^{2}$ at pre-necking stage (stage 2) being relatively low indicates the source location of $\mathrm{AE}$ events to be concentrated about the center line of the gauge region as mechanical deformation proceeds. The small angle $\mathrm{X}$-ray scattering shows that during deformation, the number of submicrocarcks increases rapidly, though there is no appearance of macroscopic craze formation on the sample surface. Thus, prior to the necking formation, AE signals may be generated on nucleation and/or with the development of submicrocracks. This mechanism of AE events in PC is similar to that in PMMA, as was proposed in our previous study. ${ }^{1}$ It should be pointed out that AE signals of detectable amplitude are produced only cooperative generation of submicrocracks and not with the formation of each submicrocrack. Nucleation of submicrocracks generally tends to concentrated in the highly stressed region, i.e. about the center line of the gauge region.

On completion of necking formation (stages 3, 4) AE characteristics change remarkably. At (the necking propagation) stage (4), the AE signals showed relatively long duration times, more than $80 \mu$ s and the value of $\sigma^{2}$ was 1.9 $\left(\mathrm{mm}^{2}\right)$. The former finding suggests that $\mathrm{AE}$ signals are generated by the durable and slow release of stored energy and the latter, that almost all AE events occur at the necking front. In such a large-scale deformation stage, the molecular chains under applied stress should cause preferential segmental motion along the direction of applied stress due to an induced unsymmetrical potential barrier, as suggested by a rate process theory. ${ }^{10}$ Thus, when molecules are drawn from the random coils in the glassy state and oriented in the direction of stress, various frictional sounds are probably produced. These sounds are considered responsible for the AE signals detected following the necking formation.

\section{CONCLUSION}

A typical ductile polymer, PC, generated 
strong burst type AE signals throughout deformation. AE characteristics, such as wave form and $\mathrm{AE}$ source distribution, changed remarkably with the formation of necking. Before necking, AE signals with duration times less than $50 \mu \mathrm{s}$, were observed randomly over the entire gauge region and after necking, AE signals were mostly generated in the necking front with duration times longer than $80 \mu \mathrm{s}$. AE signals detected before the necking formation may possibly be originated on formation of submicrocracks, while those during necking propagation may be associated with molecular chain slipping at the necking front.

Acknowledgments. This work was supported in part by a Grant-in-Aid for Scientific Research from the Ministry of Education, Science and Culture of Japan. We are also grateful to Mr. Y. Sakurada for his technical assistance.

\section{REFERENCES}

1. S. Shichijyo, S. Shirouzu, S. Taki, K. Matsushige, and T. Takemura, Jpn. J. Appl. Phys., 22, 1315 (1983).

2. I. Grabec and A. Peterlin, J. Polym. Sci., Polym. Phys. Ed., 14, 651 (1976).

3. A. Peterlin, Adv. Chem. Ser., 174, 15 (1979).

4. T. Nishiura, T. Joh, S. Okuda, and M. Miki, Zairyo, 32, 88 (1983).

5. M. Ja. Tutans and Yu. S. Urzhumtsev, Polym. Mech., 7, 377 (1971).

6. V. S. Kuksenko and V. P. Tamuzs, "Fracture Micromechanics of Polymer Materials," Martinus Nijhoff Publishers, Haugue, 1981.

7. D. Betteridge, J. V. Gridland, T. Lilley, N. R. Shoko, M. E. A. Cudby, and D. G. M. Wood, Polymer, 23, 178 (1982).

8. E. Roeder and H. A. Crostack, Kunststoffe, 67, 454 (1977).

9. J. Sauer and C. Hsiao, Trans. ASME, 75, 895 (1953).

10. R. Robertson, J. Appl. Polym. Sci., 7, 443 (1963). 